\title{
Ergonomics in the Industry 4.0: Virtual and Augmented Reality
} Luis Miguel Munoz*

Department of Automatic Control and Computer Engineering, Universitat Politecnica de Catalunya, Spain

*Corresponding author: Luis Miguel Munoz, Department of Automatic Control and Computer Engineering, Universitat Politecnica de Catalunya, Spain, Tel: 938967231; E-mail: luis.miguel.munoz@upc.edu

Received date: August 14, 2018; Accepted date: August 16, 2018; Published date: August 24, 2018

Copyright: $\odot 2018$ Munoz LM. This is an open-access article distributed under the terms of the Creative Commons Attribution License, which permits unrestricted use, distribution, and reproduction in any medium, provided the original author and source are credited.

Citation: Munoz LM (2018) Ergonomic in the Industry 4.0: Virtual and Augmented Reality. J Ergonomics 8:e181. Doi: 10.4172/2165-7556.1000e181

\section{Editorial}

The environment of Industry 4.0 is producing a change in the way workers and machines coexist $[1,2]$. One of the key aspects of Industry 4.0 is the digitalization of processes, leading to an approach between physical systems and information systems. Having real-time information on the processes in a modern industry can predict failures, and anticipate changes in market demands. Computer-aided maintenance and repairing reduces process downtime by facilitating and reducing the workload of maintenance operators.

Within the digitalization of the industry appears the concept of virtual factory, which leads to virtual and augmented reality technologies. In virtual reality (VR) we have a fully digitized world of industry over which the user can navigate and interact. In augmented reality (AR), digital information is superimposed on real images obtained directly or indirectly, in order to enrich the information that reaches the observer [3]. In aspects of maintenance and assembly, the operator observes information about the systems, their references, operating states, guidelines for assembly/disassembly of parts, etc.

The potential benefits of AR on operators are [4]: Reduction of movement of head and eyes, reduction of commutation of contexts, reduction of repairing time, collaboration in real time, historical documentation, complementary training, among others.

Although AR and VR technologies can be projected on computers or tablets, head-mounted displays and stereoscopic glasses are more practical for workers as it allows hands-free use and a more realistic and immersive point of view. This raises ergonomic issues that must be resolved. The effects of AR are similar to those of VR when similar technologies are used. These effects $[5,6]$ can be physical, physiological and psychological.

The physical effects can be postural, repetitive, due to the weight and adjustment of the device, due to hygiene, as well as those due to the effects of the immersion (collisions with the environment).
On the other hand, the physiological effects are mainly those that affect the vision system due to the use of stereoscopic devices, such as the dissociation of accommodation and convergence, the relationship between the inter-screen distance, the inter-ocular distance of the lenses and the inter-pupillary distance of the user. Some of these effects are solved with the inclusion of focusing elements and adjustment of the optical system. Another effect that has been observed is the socalled simulator sickness that is known in the literature in the use of pilots subjected to flight simulators, some of its effects are apathy, fatigue, and disorientation.

As for the psychological effects, in rare cases there may be episodes of hallucinations, dissociation, anxiety and claustrophobia.

Most of the cited effects occur with a prolonged use, however we must not leave out the benefits for the long-term user in its quality and workload, which should establish guidelines for its use for a good ergonomics. Although there is a long way to the widespread use of these technologies in the industrial environment, work must still be done to obtain standards and regulations for their design and use [6].

\section{References}

1. Munoz LM (2017) Ergonomics in the Industry 4.0: Collaborative Robots. J Ergonomics 7: e173.

2. Munoz LM (2018) Ergonomics in the Industry 4.0: Exoskeletons. J Ergonomics 8: e176.

3. Roitman L, Shrager J, Winograd T (2017) A Comparative Analysis of Augmented Reality Technologies and their Marketability in the Consumer Electronics Segment. J Biosens Bioelectron 8: 236.

4. CostelloPJ (1997) Health and Safety Issues associated with Virtual Reality -A review of Current Literature. Advanced VR Research Centre.

5. Nichols S, Patel H (2002) Health and safety implications of virtual reality: a review of empirical evidence. Appl Ergonom 33: 251-271.

6. Ritsos PD, Ritsos DP, Gougoulis AS (2011) Standards for Augmented Reality: a User Experience perspective. Synthetic Toys. 